\title{
Location matters: the endoplasmic reticulum and protein trafficking in dendrites
}

\author{
Omar A. Ramírez ${ }^{1,2}$, Steffen Härtel 2,3 and Andrés Couve ${ }^{1,3^{*}}$ \\ ${ }^{1}$ Program of Physiology and Biophysics, Institute of Biomedical Sciences, Universidad de Chile, Santiago, Chile. \\ 2 Laboratory of Scientific Image Analysis (SCIAN-Lab), Program of Anatomy and Developmental Biology, Institute of Biomedical Sciences, Universidad de Chile, Santiago, Chile. \\ ${ }^{3}$ Nucleus of Neural Morphogenesis (NEMO), Faculty of Medicine, Universidad de Chile, Santiago, Chile.
}

\begin{abstract}
Neurons are highly polarized, but the trafficking mechanisms that operate in these cells and the topological organization of their secretory organelles are still poorly understood. Particularly incipient is our knowledge of the role of the neuronal endoplasmic reticulum. Here we review the current understanding of the endoplasmic reticulum in neurons, its structure, composition, dendritic distribution and dynamics. We also focus on the trafficking of proteins through the dendritic endoplasmic reticulum, emphasizing the relevance of transport, retention, assembly of multi-subunit protein complexes and export. We additionally discuss the roles of the dendritic endoplasmic reticulum in synaptic plasticity.
\end{abstract}

Running title: structure and function of the dendritic endoplasmic reticulum. Key terms: endoplasmic reticulum, protein trafficking, dendrites, neuron.

\section{INTRODUCTION}

The transport of newly synthesized membrane lipids and proteins to distal regions in dendrites and axons is essential for neuronal function. Despite the obvious spatial and geometrical constrains that condition these biosynthetic events, the structure of secretory organelles and the trafficking mechanisms in the neuron's highly polarized morphology are only beginning to emerge (Pierce et al., 2001; Kennedy and Ehlers, 2006). For example, endosomal compartments bearing Rab11 are present in dendrites and dendritic spines where they carry out recycling functions to and from the plasma membrane (Wang et al., 2008). Likewise, functionally competent Golgi outposts containing galactosyltransferase and GM130 have been observed in dendrites (Kennedy and Ehlers, 2006). Dendrite growth and identity may depend on these structures because Golgi outposts regulate dendritic branching and morphology (Horton et al., 2005; Ye et al., 2007).

In contrast to recent advances in the understanding of late secretory organelles the structure of the endoplasmic reticulum (ER) in dendrites, and its role in de novo synthesis and trafficking of neuronal proteins have received substantially less attention. This is surprising considering the potential role of the dendritic ER in synaptic plasticity, for instance through the control of transport, assembly and export of neurotransmitter receptors in the proximity of synapses. Here we review the dynamic structure of the ER in dendrites, its role in the anterograde trafficking of neuronal proteins and its contribution to synaptic plasticity.

The structure of the endoplasmic reticulum in dendrites

The ER in neurons is constituted by i) a continuous irregular network of thin tubules, ii) flat widened regions of variable diameter, and possibly iii) a vesicular component.
Together this structure is appropriately referred to as a bumpy network (Cooney et al., 2002; Jones et al., 2008). The ER connects the soma with the entire dendritic arbor, including distal dendrites, and the axon. The ER is predominantly located in the cortex of the dendritic cytoplasm with thin branches traversing the opposite side and abundant surface area neighboring the plasma membrane (Cooney et al., 2002; Krijnse-Locker et al., 1995).

The ER in the soma and the proximal somatodendritic compartment is ribosome enriched, corresponding to rough ER (RER), while the ER distributed in distal dendrites bears only few sparse ribosomes and its mostly smooth ER (SER). The continuity of the SER is critical for the propagation of $\mathrm{Ca}^{2+}$ signals, and for the synthesis and transport of lipids and proteins (Horton and Ehlers, 2003; Petersen and Verkhratsky, 2007). The SER volume represents a small fraction of a dendrite $(3,2 \%$ of dendrite volume) and does not change with age, despite the fact that its surface increases 1,7 fold from postnatal day 15 to adult in mice (Cooney et al., 2002). The SER is also present in the head or neck of $20 \%$ of adult dendritic spines, where it occasionally associates to a prominent specialization called the spine apparatus (SA) that can be identified by labeling the actin-binding protein synaptopodin (Cooney et al., 2002; Vlachos et al., 2009).

The area occupied by the dendritic ER correlates with the number of spines or synapses occurring in the corresponding segment (Spacek and Harris, 1997). In agreement with these findings the ER is found in proximity to the plasma membrane in presynaptic and postsynaptic specializations, where it often associates with cytoskeletal elements. The proximity of the ER to the cell surface is also seen at adherent puncta at non synaptic sites (Spacek and Harris, 1998). Although a significant subset of spines contains stable ER ( $\sim 4$ days), the ER present in other spines is highly dynamic and shows a high degree of turnover (Toresson and Grant, 2005). These results may help 
explain the variation in $\mathrm{Ca}^{2+}$ spike magnitude or localized protein synthesis and trafficking in individual spines (Toresson and Grant, 2005).

Features recently uncovered in other eukaryotic cell types have begun to provide insights into the structure of the ER in neurons. For example, reticulons are a family of eukaryotic proteins associated to ER membranes that are responsible for keeping the ER's tubular shape (Sparkes et al., 2009; Voeltz et al., 2006). Interestingly, reticulon proteins RTN2B and RTN3 are distributed along neurites (Hu et al., 2007; Liu et al., 2008), and overexpression of RTN3 causes aggregation and neuritic dystrophy (Hu et al., 2007). In addition, a dynaminlike GTPase called atlastin-1 interacts directly with reticulon proteins promoting fusion and the formation of the tubular ER network. Overexpression of a GTPase-deficient atlastin-1 in mammalian cells produces long unbranched ER tubules. Thus, atlastin-1 drives ER membrane fusion in a GTPase dependent manner (Hu et al., 2009). A recent study demonstrates that atlastin-1 depletion causes the fragmentation of the neuronal ER in Drosophila (Orso et al., 2009). The fact that atlastin-1 mutations cause hereditary spastic paraplegia (Salinas et al., 2008), indicates that loss of ER shape has pathological consequences. However, the precise roles of atlastins and other structural components of the ER in mammalian dendrites await clarification.

The dendritic ER is not a uniform structure but rather an irregular tubular network with a variety of microdomains. For example, in spite of its continuous structure, the ER has a heterogeneous distribution of inositol 1,4,5-trisphosphate receptors, ryanodine receptors and calreticulin (Bannai et al., 2004; Copray et al., 1996; Vlachos et al., 2009). The differential localization of proteins related to $\mathrm{Ca}^{2+}$ signaling is an important factor for the spatial regulation of $\mathrm{Ca}^{2+}$ release, allowing the control of $\mathrm{Ca}^{2+}$ transport in local domains (Petersen and Verkhratsky, 2007). The heterogeneous distribution of proteins in microdomains of the ER is not unique to neurons and has been explored thoroughly in other cell types. For instance, calsequestrin, is segregated in filamentous matrices of dense core, specifically in terminal cisternae of the sarcoplasmic reticulum in muscle cells. This differential distribution occurs by a process called condensation, which is different from aggregation due to disulfide bonds of misfolded proteins that are delivered for degradation (Gatti et al., 2001). In dendrites the existence and function of ER microdomains need to be further investigated in the context of an actively shaped network that supports high connectivity, segregated functions and dynamic changes.

The structure of the ER in dendrites is regulated by extracellular signals. For example, activation of NMDARs disrupts the continuity of the ER (Kucharz et al., 2009). The fission of the dendritic ER caused by NMDARs is reversible, suggesting that it is a physiologically relevant phenomenon. Neuronal ER fission may protect the neuron against propagation of excessive $\mathrm{Ca}^{2+}$ release under pathological conditions, such as ischemia. Alternatively discontinuity may favor local trafficking from the ER, ensuring a local supply of proteins to the synapse.

Recently, we have addressed the issue of ER shape and glutamate receptor activation. We evaluated changes in the ER by confocal microscopy and image processing. As expected, under control conditions the dendritic ER occupied the dendrite along its entire length, and was visualized as a heterogeneous structure (Figure 1A, upper panel). We measured the number and volume of discrete ER compartments. The resulting numbers were distributed in increasing volume intervals in a normalized frequency plot (Figure 1B), and showed that large structures $\left(>15 \mu \mathrm{m}^{3}\right)$ contained the main proportion of the dendritic ER volume (Figure 1A upper panel red, and Figure 1B solid red bar). Accordingly, Figure 1A showed a single red structure, which most likely corresponded to a continuous ER network. Importantly, the ER was fragmented upon glutamate receptor activation (Figure 1A, lower panel). In agreement with these findings the volume of the larger structures diminished significantly after glutamate receptor activation (Figure 1A lower panel red, green and yellow, and Figure 1B, dashed bars). This analysis confirms that the dendritic ER can change its structure from a largely continuous network to a fragmented collection of intermediate compartments in response to glutamate stimulation. More importantly, it provides a powerful tool to examine local changes in the structure of the ER in response to external stimuli.

Trafficking of membrane proteins through the dendritic ER: retention, assembly and export

Properly folded proteins are concentrated at ER exit sites (ERES) that are located throughout the ER in the cell body and dendritic arbor. Dendritic ERES (dERES) have been identified by detecting components of the coat protein complex II (COPII), such as the soluble GTPase Sar1, Sec23/24 and Sec13/31 (Aridor et al., 2004). Importantly, the functional relevance of dERES is supported by the existence of local post-ER secretory compartments. For example, it has been documented in dendrites that cargo exits the ER to enter the ER-Golgi intermediate compartment (ERGIC) (Gardiol et al., 1999). Accordingly, specific ERGIC markers such as the GTPase Rab1 and the oligomeric type I membrane lectin ERGIC-53 have been detected in distal dendrites and spines (Pierce et al., 2001). Functional evidence for the dendritic ERGIC has also been reported. In a recent study Farhan and coworkers demonstrated that a motif constituted by three hydrophobic residues of the GABA transporter 1 (GAT1) (VMI 569-571) is required for release from the ER-Golgi intermediate compartment, and that replacement of these aminoacids results in the accumulation of structures containing GAT1 in the soma and neurites of hippocampal neurons (Farhan et al., 2008).

The presence of dERES suggests that tight mechanisms are in place to control the assembly and export of dendritic proteins, and therefore stress the importance of ER checkpoints and transient ER retention. Multiple examples illustrate the existence of a variety of molecular mechanisms to control multimeric assembly, ER export and plasma membrane availability across very distinct protein families. Together with the utilization of similar mechanisms in different species they confirm the evolutionary pressure to maintain ER trafficking checkpoints in neurons. However, it still remains to be determined whether all these control mechanisms operate in distal dendrites, how the molecular machineries connect ER export and neuronal geometry, and to what extent these potentially local mechanisms contribute to modulate the surface availability of dendritic proteins. 
The best described ER retention signals, KDEL and di-lysine $(\mathrm{K}(\mathrm{X}) \mathrm{KXX})$ for luminal and membrane proteins respectively, can be found in resident ER chaperones (e.g. BiP and calreticulin) or enzymes (e.g. signal peptidase). The KDEL sequence functions as a retrieval/recycling signal that is recognized by KDEL receptors located at post-ER compartments that maintain an enrichment of the protein pool in the ER. The retrieval mediated by the di-lysine motif operates in conjunction with COPI coated vesicles (Murshid and Presley, 2004). In neurons, many ion channels and neurotransmitter receptors are multi-subunit proteins that require assembly to exit the ER and avoid degradation. Thus, ER retention has evolved as a mechanism to prevent unassembled or misfolded multimers to reach the plasma membrane avoiding harmful effects on neuronal function. Interestingly, sequences different from KDEL and di-lysine control the trafficking of dendritic proteins to the plasma membrane.

Arginine-based ER retention signals are present in several neuronal proteins that function outside of the ER. Masking of arginine-based ER retention signals through heteromerization is a requisite for ER export and appearance of functional receptors at the plasma membrane. For instance, subunits of the ATP-sensitive potassium $\left(\mathrm{K}_{\text {ATP }}\right)$ channels (Kir6.1/2 and SUR1), splice variants of the NMDA receptor (NMDAR) subunits (NR1-1) and $G_{A B A}$ receptor $\left(G A B A_{B} R\right)$ subunits $\left(\mathrm{GABA}_{\mathrm{B}} \mathrm{R} 1\right)$ contain variants of the arginine-based motif in their intracellular C-terminal domains (Michelsen et al., 2005). Arginine-based signals are exposed to the cytosolic milieu in incompletely assembled complexes and become masked when dimers or multimers are properly constituted (Michelsen et al., 2005). A clear example of the functional relevance of the arginine-based retention signals in the assembly and forward trafficking of neuronal proteins is provided by $G_{A B A}$ Rs. The $G_{A B A} R 1$ subunit contains a LRSRR type sequence in its C-terminal intracellular domain that functions as an ER retention motif (Couve et al., 1998; Margeta-Mitrovic et al., 2000). The ER retention sequence of $G A B A_{B} R 1$ is masked upon assembly with $\mathrm{GABA}_{B} \mathrm{R} 2$ resulting in ER export of the functional heteromeric receptor and its subsequent insertion at the plasma membrane (Margeta-Mitrovic et al., 2000). Similarly, subunits of the $K_{\text {ATP }}$ channel contain RKR type retention sequences that are masked prior to ER exit (Zerangue et al., 1999). A further example is provided by NMDARs, where the NR1-1 and NR2B subunits contain several ER retention signals, including a conventional RRR type sequence in the C-terminus of NR1-1 and other sequences in the third transmembrane segments of NR1-1 and NR2B subunits (Horak et al., 2008).

Other neurotransmitter receptors contain retention sequences and mechanisms of a different kind. Nicotinic acetylcholine receptors (nAChRs) are pentameric ligand gated ion channels, which exist in multiple subunit stoichiometries and are localized in distinct microdomains at the plasma membrane (Drenan et al., 2008). It is thought that neurons contain large intracellular pools of assembled, functional nicotinic receptors, which may provide cells with reserve pools to rapidly up-regulate nicotinic responses to ligands. As a general rule, exit from the ER is an important checkpoint regulating surface expression of nAChRs. Correct subunit assembly favors ER exit and surface trafficking of functional receptors by hindering a $\mathrm{PL}(\mathrm{Y} / \mathrm{F})(\mathrm{F} / \mathrm{Y}) \mathrm{XXN}$ motif, which is buried in the pentamer. As has been suggested for other multimeric membrane proteins the exposed sequence in the unassembled subunits avoids delivery to the plasma membrane and promotes subunit degradation (Wang et al., 2008). Other examples of ER retention-retrieval are provided
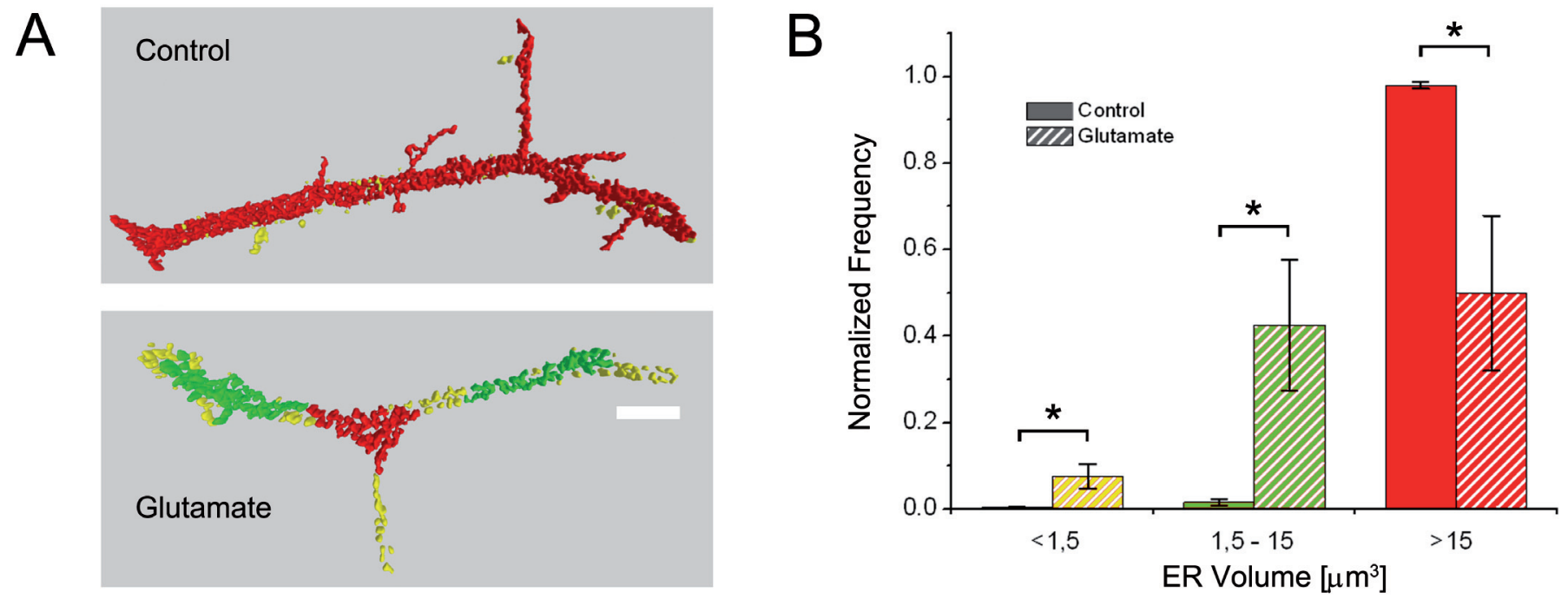

Figure 1: Glutamate receptor activation induces ER fragmentation. A) 7-9 days in vitro cultured hippocampal neurons were transfected with the fusion protein mRFP-KDEL. $24 \mathrm{~h}$ after transfection neurons were left unstimulated (upper panel) or treated with $100 \mu \mathrm{M}$ glutamate for $30 \mathrm{~min}$ (lower panel). Confocal z-stack fluorescent images of dendrites were obtained every $0.3 \mu \mathrm{m}$, deconvolved by Huygens Scripting software (Scientific Volume Imaging, Hilversum, Netherlands), segmented, 3D-rendered and quantified with image-processing routines developed in our laboratory based on Interactive Data Language (ITT, Boulder, CO). The volume of discrete ER structures was measured. Volume categories were color coded (yellow: $<1.5 \mu \mathrm{m}^{3}$; green: $1.5-15 \mu \mathrm{m}^{3}$; red: $>15 \mu \mathrm{m}^{3}$ ). Images show representative dendrites. Scale bar $5 \mu \mathrm{m}$. B) The normalized frequency histogram was calculated on the basis of 7 control and 7 glutamate stimulated dendrites (Solid and dashed bars respectively). Bars are mean \pm s.e.m. $\left({ }^{*} \mathrm{p}<0.01, \mathrm{t}\right.$-test). 
by an aspartate residue at the boundary of the M3-M4 loop and M4 domain of ionotropic $\mathrm{GABA}_{\mathrm{A}}$ receptors (Lo et al., 2008) and by an ER retention signal (CRAR) located in the first cytoplasmic loop of one subunit of serotonin receptors, namely 5-HT3B (Boyd et al., 2003; Connolly, 2008).

Protein trafficking is regulated by ER retention and also by the efficiency of ER export and forward trafficking signals. For instance the efficiency of plasma membrane delivery varies for different subunits of inwardly rectifying potassium channels. ER export sequences in the C-termini of Kir1.1 (VLS and EXD) and Kir2.1 (FCYENE) differentially control the export of potassium channels from the ER (Ma et al., 2001). Additionally, the excitatory amino acid transporter EAAC1 that regulates extracellular glutamate concentration, is also regulated at the ER. EAAC1 is retained in the ER by its associate protein GTRAP3-18, but the interaction with RTN2B, a reticulon family member promotes EAAC1 ER exit (Liu et al., 2008), uncovering a new function for ER structural components in ER trafficking.

\section{Dendritic ER transport and export as a mechanism to reach distal sites}

Two models have been put forward to explain protein trafficking in dendrites. A first one relates to the canonical view of a centralized Golgi compartment that generates mature proteins, which are sorted by post-Golgi secretory vesicles that travel long distances towards the plasma membrane. The model proposes that post-Golgi vesicles move along microtubules assisted by molecular motors. Unloading of vesicles from microtubules occur in the vicinity of synapses in a $\mathrm{Ca}^{2+}$-dependent manner (Guillaud et al., 2008). Some neurotransmitter receptors may follow the canonical route using the somatic ER, the somatic Golgi apparatus and long-haul post-Golgi vesicles (Figure 2). For example, a conventional kinesin and glutamate-receptor-interacting protein1/AMPAR-binding (GRIP1/ABP) mediate the dendritic transport of AMPARs, presumably on dendritic post-Golgi vesicles, before insertion at the plasma membrane (Setou et al., 2002). Once in the proximity of the post synaptic density rapid changes in cell surface abundance are controlled by entry and exit of recycling endosomes into the spine in a myosin $\mathrm{Vb}$ dependent manner (Wang, et al., 2008). Reports on NMDAR and GlyRs also support a canonical pathway. For NMDARs the movement of vesicular structures has also been directly visualized in vitro (Setou et al., 2000). Newly synthesized GlyRs a1 subunits insert preferentially in the somatic and proximal dendritic plasma membrane and later diffuse along the plasma membrane where they are retained in synapses at distal dendrites (Rosenberg et al., 2001). Combined, these results support the use of somatic secretory organelles and post-Golgi transport vesicles for dendritic transport.

Recently, a more complex scenario of secretory organelles has supported an alternative model (Pierce et al., 2001; Vlachos et al., 2009). The model rests on the existence of a local secretory route in which the functional ER is distributed throughout the neuron and releases cargo from dERES towards Golgi outposts, including local sorting stations in the generation and maintenance of neuronal microdomains (Horton and Ehlers, 2003) (Figure 2). We now focus on this mode of trafficking and illustrate the role of the dendritic ER in the transport and trafficking of multimeric proteins to synaptic, extrasynaptic or perisynaptic sites in dendrites.
First, although some studies on NMDARs have suggested a canonical transport mechanism (Setou et al., 2000; Guillaud et al., 2003) current evidence strongly supports an alternative dendritic trafficking mode. NR1 subunits concentrate at dERES as revealed by their enrichment in Sar1 positive puncta (Aridor et al., 2004) indicating that dERES have a functional role in regulating the local trafficking and synaptic availability of NMDARs. Supporting these results a recent study shows that NMDARs concentrate in the dendritic ER and use unconventional Golgi outposts before inserting at the plasma membrane (Jeyifous et al., 2009).

$\mathrm{GABA}_{\mathrm{B}}$ Rs provide another example. Functional $\mathrm{GABA}_{\mathrm{B}} \mathrm{R}$ heterodimers localize to the plasma membrane, but dendritic intracellular compartments contain abundant segregated subunits (Gonchar et al., 2001; Ramirez et al., 2009) (Figure 3). Blocking ER exit with a Sar1 dominant negative results in the accumulation of heterodimers in the somato-dendritic ER indicating that newly assembled receptors rapidly traffic from dERES to the plasma membrane (Ramirez et al., 2009) (Figure 3). Whether $\mathrm{GABA}_{\mathrm{B}}$ Rs traffic via central or local Golgi outposts, and whether they reach the plasma membrane in the somatic region and then diffuse laterally along the plasma membrane or insert locally in the vicinity of functional sites in dendrites are still unanswered questions.

Finally, serotonin receptors provide an interesting relationship between trafficking and dendritic transport. The

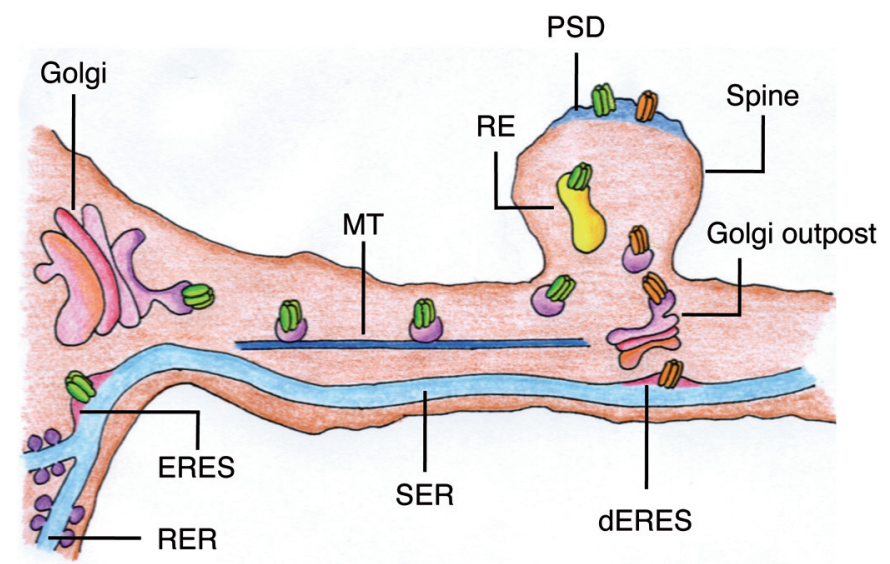

Figure 2: Two models for protein trafficking in dendrites: (i) The drawing outlines the canonical view of a centralized Golgi compartment and sorting by means of post-Golgi secretory vesicles. These travel along microtubules assisted by molecular motors and unload in vicinity of synapses in a $\mathrm{Ca}^{2+}$-dependent manner for subsequent rapid entry and exit of recycling endosomes into spines. Receptors using this mode of trafficking are shown in green. (ii) The scheme also shows an alternative model considering a more complex scenario of secretory organelles. The rough ER (RER) is mainly somatic but the smooth ER (SER) is distributed throughout the neuron and releases cargo from dERES towards Golgi outposts. Receptors using this mode of trafficking are shown in orange. They exit the ER using dERES, move towards Golgi outposts, and use shorthaul post-Golgi vesicles prior to membrane insertion. RER (rough endoplasmic reticulum), SER (smooth endoplasmic reticulum), ERES (endoplasmic reticulum exit sites), dERES (dendritic endoplasmic reticulum exit sites), MT (microtubules), RE (recycling endosome), PSD (postsynaptic density). 
serotonin $5-\mathrm{HT}_{1 \mathrm{~A}}$ receptor depends on its C-terminus to reach distal dendrites in cultured hippocampal neurons. It was recently described that $5-\mathrm{HT}_{1 \mathrm{~A}}$ also requires a protein partner, Yif1B, to acquire distal dendritic expression. Yif1B is an ERGIC associated protein and $5-\mathrm{HT}_{1 \mathrm{~A}}$ receptors redistribute to the somatic and proximal dendritic compartment when Yif1B is removed, reflecting a potential contribution to functional modulation (Carrel et al., 2008). Therefore, ER to Golgi transport is needed for the long distance mobility of $5-\mathrm{HT}_{1 \mathrm{~A}}$ receptors. Combined, the results on NMDARs, $\mathrm{GABA}_{B}$ Rs and $5-\mathrm{HT}_{1 \mathrm{~A}}$ receptors suggest that the dendritic ER provides a non canonical pathway of protein trafficking in dendrites.

Uncovering the mechanisms responsible for the transport or mobility of neurotransmitter receptors along the dendritic ER membrane is essential to fully understand the contribution of local protein trafficking to synaptic plasticity. Rapid AMPAR trafficking between the plasma membrane and recycling endosomes argues against protein synthesis regulating short-term changes in receptor availability (Newpher and Ehlers, 2008; Wang, et al., 2008). This evidence suggests that the dendritic ER may function as a storage compartment prior to the endosomal system and that similar reservoir mechanisms may operate in other polarized cells (SomselRodman and Wandinger-Ness, 2000). However, little is known about the transport of neurotransmitter receptors along the dendritic ER membrane. Diffusion may not be sufficient to drive neurotransmitter receptors outwards, but mobility may be assisted by molecular motors. For example, dendritic trafficking of NMDARs is dependent on the scaffold proteins CASK and SAP97, which form complexes with motor proteins and sort receptors to ER and Golgi outposts for plasma

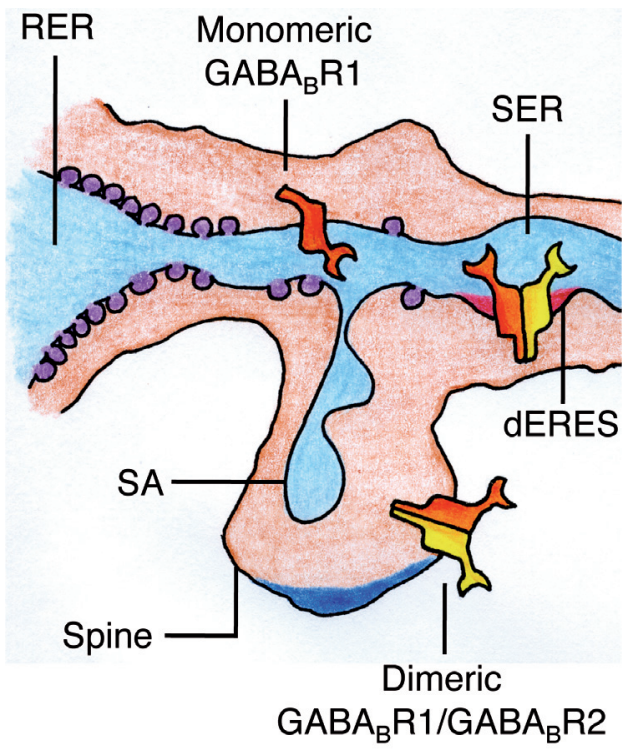

Figure 3: Schematic view of the mobility and intracellular trafficking of $G A B A_{B} R s$ receptors in dendrites. $G A B A_{B} R 1$ may be transported along the ER membrane before heterodimerization (monomeric $\left.G A B A_{B} R 1\right)$. Heterodimerization and $E R$ exit of $\mathrm{GABA}_{\mathrm{B}} \mathrm{Rs}$ occur in dERES. The ER-Golgi transport mechanism for $G_{A B A} R S$ is still unknown. RER (rough endoplasmic reticulum), SER (smooth endoplasmic reticulum), dERES (dendritic endoplasmic reticulum exit sites), SA (spine apparatus). membrane delivery (Jeyifous et al., 2009). Likewise, a dominant negative KIF5 alters the intracellular localization of ER retained wild-type $\mathrm{GABA}_{B} \mathrm{R} 1$ but not of a subunit with mutations in the ER retention motif (Vidal et al., 2007). The role of molecular motors in ER transport along dendrites is further supported by evidence indicating that resident proteins of the ER move from the soma to dendrites in a microtubule and kinesin-dependent manner (Bannai et al., 2004; Copray et al., 1996; Vlachos et al., 2009). Whether the mobile compartment is a vesicular ER component or a more complex dynamic structure, such as large protein complexes moving through dilated or bulged tubular ER membranes, remains to be determined.

It is a matter of debate whether ER transport and distal ER export constitute general mechanisms or if they are only used by a subset of proteins. However, accumulated observations indicate that multiple trafficking mechanisms coexist in neurons to accommodate their specialized morphological demands.

\section{Dendritic ER trafficking and plasticity}

The current structural evidence provides a morphological basis for understanding the role of the ER in synaptic function and plasticity. The fact that the ER is highly dynamic and that is located near inhibitory postsynaptic sites together with the presence of the SA in some dendritic spines suggest that it plays a critical role in synaptic transmission. For example, it has been shown that the ER can enter and exit dendritic spines over the time course of hours (Toresson and Grant, 2005). The presence of the ER in a spine may depend on physical interactions between proteins of the ER membrane and the plasmalemma ( $\mathrm{Ng}$ and Toresson, 2008). In addition, microtubule plus end binding proteins enter spines providing a cytoskeletal link for entry and exit of the ER from dendritic spines and contributing to modulate spine morphology (Jaworski et al., 2009).

It has been firmly established that several extracellular signals impact ER function. For instance, NMDA treatments stimulate $\mathrm{Ca}^{2+}$ release from intracellular stores (Emptage et al., 1999). However, little is known of the direct impact of extracellular signals in the trafficking of newly synthesized proteins through the ER. Importantly, the control of ER exit of NMDARs in the proximity of synaptic sites is regulated by activity supporting a relationship between dendritic ER export and synaptic activity (Aridor et al., 2004; Jeyifous et al., 2009). As stated above, whether newly exported proteins directly contribute to modulate receptor availability or whether they serve to replenish an endosomal compartment remains to be studied (Wang et al., 2008).

\section{Future directions}

The concepts and observations discussed in the review indicate that many new avenues of research remain open to clarify the structure and dynamics of the ER in dendrites. They strongly suggest that local control of ER function and structure in response to neuronal activity needs to be further investigated. They also stress the need to investigate whether, in reaction, changes in ER structure generate local responses that contribute to modulate neuronal function. They also invite to examine the role of ER trafficking in the axon. Interestingly, ERES were recently detected in the axon (Aridor and Fish, 
2009). Thus, the field requires a revised framework that takes into account ER trafficking in the context of a complex neuronal geometry.

\section{ACKNOWLEDGMENTS}

OAR funded by FONDECYT 3110157 SH funded by FONDECYT 1090246. AC funded by FONDECYT 1100137. OAR, SH and AC funded by ICM P07-048-F.

\section{REFERENCES}

ARIDOR M, FISH KN (2009) Selective targeting of ER exit sites supports axon development. Traffic 10: 1669-1684

ARIDOR M, GUZIK AK, BIELLI A, FISH KN (2004) Endoplasmic reticulum export site formation and function in dendrites. J Neurosci 24: 3770-3776

BANNAI H, INOUE T, NAKAYAMA T, HATTORI M, MIKOSHIBA K (2004) Kinesin dependent, rapid, bi-directional transport of ER subcompartment in dendrites of hippocampal neurons. J Cell Sci 117: 163-175

BOYD GW, DOWARD AI, KIRKNESS EF, MILLAR NS, CONNOLLY CN (2003) Cell surface expression of 5-hydroxytryptamine type 3 receptors is controlled by an endoplasmic reticulum retention signal. J Biol Chem 278: $27681-27687$

CARREL D, MASSON J, AL AWABDH S, CAPRA C B, LENKEI Z, HAMON M, EMERIT MB, DARMON M (2008) Targeting of the 5-HT1A serotonin receptor to neuronal dendrites is mediated by Yif1B. J Neurosci 28: 8063-8073

CONNOLLY CN (2008) Trafficking of 5-HT(3) and GABA(A) receptors (Review). Mol Membr Biol 25: 293-301

COONEY JR, HURLBURT JL, SELIG DK, HARRIS KM, FIALA JC (2002) Endosomal compartments serve multiple hippocampal dendritic spines from a widespread rather than a local store of recycling membrane. J Neurosci 22: 2215-2224

COPRAY JC, LIEM RS, KERNELL D (1996) Calreticulin expression in spinal motoneurons of the rat. J Chem Neuroanat 11: 57-65

COUVE A, FILIPPOV AK, CONNOLLY CN, BETTLER B, BROWN DA, MOSS SJ (1998) Intracellular retention of recombinant GABAB receptors. J Biol Chem 273: 26361-26367

DRENAN RM, NASHMI $R$, IMOUKHUEDE P, JUST H, MCKINNEY $S$, LESTER HA (2008) Subcellular trafficking, pentameric assembly, and subunit stoichiometry of neuronal nicotinic acetylcholine receptors containing fluorescently labeled alpha6 and beta3 subunits. Mol Pharmacol 73: 27-41

EMPTAGE N, BLISS TV, FINE A (1999) Single synaptic events evoke NMDA receptor-mediated release of calcium from internal stores in hippocampal dendritic spines. Neuron 22: 115-124

FARHAN H, REITERER V, KRIZ A, HAURI HP, PAVELKA M, SITTE HH, FREISSMUTH M (2008) Signal-dependent export of GABA transporter 1 from the ER-Golgi intermediate compartment is specified by a C-terminal motif. J Cell Sci 121: 753-761

GARDIOL A, RACCA C, TRILLER A (1999) Dendritic and postsynaptic protein synthetic machinery. J Neurosci 19: 168-179

GATTI G, TRIFARI S, MESAELI N, PARKER JM, MICHALAK M, MELDOLESI J (2001) Head-to-tail oligomerization of calsequestrin: a novel mechanism for heterogeneous distribution of endoplasmic reticulum luminal proteins. J Cell Biol 154: 525-534

GONCHAR Y, PANG L, MALITSCHEK B, BETTLER B, BURKHALTER A (2001) Subcellular localization of GABA(B) receptor subunits in rat visual cortex. J Comp Neurol 431: 182-197

GUILLAUD L, SETOU M, HIROKAWA N. (2003) KIF17 dynamics and regulation of NR2B trafficking in hippocampal neurons. J Neurosci. 23(1):131-40.

GUILLAUD L, WONG R, HIROKAWA N (2008) Disruption of KIF17-Mint1 interaction by CaMKII-dependent phosphorylation: a molecular model of kinesin-cargo release. Nat Cell Biol 10: 19-29

HORAK M, CHANG K, WENTHOLD RJ (2008) Masking of the endoplasmic reticulum retention signals during assembly of the NMDA receptor. J Neurosci 28: 3500-3509

HORTON AC, EHLERS MD (2003) Dual modes of endoplasmic reticulumto-Golgi transport in dendrites revealed by live-cell imaging. J Neurosci 23: 6188-6199

HORTON AC, RÁCZ B, MONSON EE, LIN AL, WEINBERG RJ, EHLERS MD, (2005) Polarized secretory trafficking directs cargo for asymmetric dendrite growth and morphogenesis. Neuron. 48:757-771.
HU J, SHIBATA Y, ZHU PP, VOSS C, RISMANCHI N, PRINZ WA, RAPOPORT TA, BLACKSTONE C (2009) A class of dynamin-like GTPases involved in the generation of the tubular ER network. Cell 138: 549-561

HU X, SHI Q, ZHOU X, HE W, YI H, YIN X, GEARING M, LEVEY A, YAN $R$ (2007) Transgenic mice overexpressing reticulon 3 develop neuritic abnormalities. Embo J 26: 2755-2767

JAWORSKI J, KAPITEIN LC, GOUVEIA SM, DORTLAND BR, WULF PS, GRIGORIEV I, CAMERA P, SPANGLER SA, DI STEFANO P, DEMMERS J, KRUGERS H, DEFILIPPI P, AKHMANOVA A, HOOGENRAAD CC (2009). Dynamic microtubules regulate dendritic spine morphology and synaptic plasticity. Neuron 61:85-100

JEYIFOUS O, WAITES CL, SPECHT CG, FUJISAWA S, SCHUBERT M, LIN EI, MARSHALL J, AOKI C, DE SILVA T, MONTGOMERY JM, GARNER CC, GREEN WN (2009) SAP97 and CASK mediate sorting of NMDA receptors through a previously unknown secretory pathway. Nat Neurosci 12: 1011-1019

JONES VC, MCKEOWN L, VERKHRATSKY A, JONES OT (2008) LV-pINKDEL: a novel lentiviral vector demonstrates the morphology, dynamics and continuity of the endoplasmic reticulum in live neurones. BMC Neurosci 9: 10

KENNEDY MJ, EHLERS MD (2006) Organelles and trafficking machinery for postsynaptic plasticity. Annu Rev Neurosci 29: 325-362

KRIJNSE-LOCKER J, PARTON RG, FULLER SD, GRIFFITHS G, DOTTI CG (1995) The organization of the endoplasmic reticulum and the intermediate compartment in cultured rat hippocampal neurons. Mol Biol Cell 6: 1315-1332

KUCHARZ K, KROGH M, NG AN, TORESSON H (2009) NMDA receptor stimulation induces reversible fission of the neuronal endoplasmic reticulum. PLoS One 4: e5250

LIU Y, VIDENSKY S, RUGGIERO AM, MAIER S, SITTE HH, ROTHSTEIN JD (2008) Reticulon RTN2B regulates trafficking and function of neuronal glutamate transporter EAAC1. J Biol Chem 283: 6561-6571

LO WY, BOTZOLAKIS EJ, TANG X, MACDONALD RL (2008) A conserved Cys-loop receptor aspartate residue in the M3-M4 cytoplasmic loop is required for GABAA receptor assembly. J Biol Chem 283: 29740-29752

MA D, ZERANGUE N, LIN YF, COLLINS A, YU M, JAN YN, JAN LY (2001) Role of ER export signals in controlling surface potassium channel numbers. Science 291: 316-319

MARGETA-MITROVIC M, JAN YN, JAN LY (2000) A trafficking checkpoint controls GABA(B) receptor heterodimerization. Neuron 27: 97-106

MICHELSEN K, YUAN H, SCHWAPPACH B (2005) Hide and run. Argininebased endoplasmic-reticulum-sorting motifs in the assembly of heteromultimeric membrane proteins. EMBO Rep 6: 717-722

MURSHID A, PRESLEY JF (2004) ER-to-Golgi transport and cytoskeletal interactions in animal cells. Cell Mol Life Sci 61: 133-145

NEWPHER TM, EHLERS MD (2008) Glutamate receptor dynamics in dendritic microdomains. Neuron. 58: 472-497

NG AN, TORESSON H (2008) Gamma-secretase and metalloproteinase activity regulate the distribution of endoplasmic reticulum to hippocampal neuron dendritic spines. FASEB J 22: 2832-2842

ORSO G, PENDIN D, LIU S, TOSETTO J, MOSS TJ, FAUST JE, MICARONI M, EGOROVA A, MARTINUZZI A, MCNEW JA, DAGA A (2009) Homotypic fusion of ER membranes requires the dynamin-like GTPase atlastin. Nature 460: 978-983

PETERSEN OH, VERKHRATSKY A (2007) Endoplasmic reticulum calcium tunnels integrate signalling in polarised cells. Cell Calcium 42: $373-378$

PIERCE JP, MAYER T, MCCARTHY JB (2001) Evidence for a satellite secretory pathway in neuronal dendritic spines. Curr Biol 11: 351-355

RAMIREZ OA, VIDAL RL, TELLO JA, VARGAS KJ, KINDLER S, HARTEL S, COUVE A (2009) Dendritic assembly of heteromeric gammaaminobutyric acid type B receptor subunits in hippocampal neurons. J Biol Chem 284: 13077-13085

ROSENBERG M, MEIER J, TRILLER A, VANNIER C (2001) Dynamics of glycine receptor insertion in the neuronal plasma membrane. J Neurosci 21: 5036-5044

SALINAS S, PROUKAKIS C, CROSBY A, WARNER TT (2008) Hereditary spastic paraplegia: clinical features and pathogenetic mechanisms. Lancet Neurol 7: 1127-1138

SETOU M, NAKAGAWA T, SEOG DH, HIROKAWA N (2000) Kinesin superfamily motor protein KIF17 and mLin-10 in NMDA receptorcontaining vesicle transport. Science 288: 1796-1802

SETOU M, SEOG DH, TANAKA Y, KANAI Y, TAKEI Y, KAWAGISHI M, HIROKAWA N (2002) Glutamate-receptor-interacting protein GRIP1 directly steers kinesin to dendrites. Nature $417: 83-87$ 
SOMSEL-RODMAN J, WANDINGER-NESS A (2000) Rab GTPases coordinate endocytosis. J Cell Sci 113 Pt 2: 183-192

SPACEK J, HARRIS KM (1997) Three-dimensional organization of smooth endoplasmic reticulum in hippocampal CA1 dendrites and dendritic spines of the immature and mature rat. J Neurosci 17: 190-203

SPACEK J, HARRIS KM (1998) Three-dimensional organization of cell adhesion junctions at synapses and dendritic spines in area CA1 of the rat hippocampus. J Comp Neurol 393: 58-68

SPARKES IA, FRIGERIO L, TOLLEY N, HAWES C (2009) The plant endoplasmic reticulum: a cell-wide web. Biochem J 423: 145-155

TORESSON H, GRANT SG (2005) Dynamic distribution of endoplasmic reticulum in hippocampal neuron dendritic spines. Eur J Neurosci 22: 1793-1798

VIDAL RL, RAMIREZ OA, SANDOVAL L, KOENIG-ROBERT R, HARTEL S, COUVE A (2007) Marlin-1 and conventional kinesin link GABAB receptors to the cytoskeleton and regulate receptor transport. Mol Cell Neurosci 35: 501-512
VLACHOS A, KORKOTIAN E, SCHONFELD E, COPANAKI E, DELLER T, SEGAL M (2009) Synaptopodin regulates plasticity of dendritic spines in hippocampal neurons. J Neurosci 29: 1017-1033

VOELTZ GK, PRINZ WA, SHIBATA Y, RIST JM, RAPOPORT TA (2006) A class of membrane proteins shaping the tubular endoplasmic reticulum. Cell 124: 573-586

WANG Z, EDWARDS JG, RILEY N, PROVANCE DW JR, KARCHER R, LI XD, DAVISON IG, IKEBE M, MERCER JA, KAUER JA, EHLERS MD (2008) Myosin $\mathrm{Vb}$ mobilizes recycling endosomes and AMPA receptors for postsynaptic plasticity. Cell 135: 535-548

YE B, ZHANG Y, SONG W, YOUNGER SH, JAN LY, JAN YN (2007) Growing dendrites and axons differ in their reliance on the secretory pathway. Cell 130: 717-729

ZERANGUE N, SCHWAPPACH B, JAN YN, JAN LY (1999) A new ER trafficking signal regulates the subunit stoichiometry of plasma membrane K(ATP) channels. Neuron 22: 537-548 